\title{
Fasting Blood Glucose Variability and Unfavorable Trajectory Patterns Are Associated with the Risk of Colorectal Cancer
}

\author{
Hyoju Jun ${ }^{1}$, Jieun Lee ${ }^{1}$, Hye Ah Lee ${ }^{2}$, Seong-Eun Kim ${ }^{3}$, Ki-Nam Shim ${ }^{3}$, Hye-Kyung Jung ${ }^{3}$, Sung-Ae Jung ${ }^{3}$, and \\ Chang Mo Moon ${ }^{3,4}$ \\ ${ }^{1}$ Department of Medicine, Ewha Womans University College of Medicine, ${ }^{2}$ Clinical Trial Center, Ewha Womans University Mokdong \\ Hospital, ${ }^{3}$ Department of Internal Medicine and ${ }^{4}$ Inflammation-Cancer Microenvironment Research Center, Ewha Womans University \\ College of Medicine, Seoul, Korea
}

\begin{abstract}
Article Info
Received February 1, 2021

Revised May 24, 2021

Accepted June 29, 2021

Published online October 1, 2021

\section{Corresponding Author}

Chang Mo Moon

ORCID https://orcid.org/0000-0003-2550-913X

E-mail mooncm27@ewha.ac.kr

Hye Ah Lee

ORCID https://orcid.org/0000-0002-4051-0350

E-mail khyeah@naver.com
\end{abstract}

Hyoju Jun and Jieun Lee contributed equally to this study as first authors.

\begin{abstract}
Background/Aims: The relationship between fasting blood glucose (FBG) variability and colorectal cancer (CRC) remains ill-defined. This study aimed to evaluate the association of FBG variability with $\mathrm{CRC}$ risk in the healthy population without overt diabetes.

Methods: In the data from the Korean National Health Insurance Service-Health Screening Cohort, we included individuals examined by FBG testing at least 3 times between 2002 and 2007 . FBG variability was calculated using standard deviation (SD) and coefficient of variation (CV).

Results: Regarding FBG variability, an increase in the quintile of SD or CV was independently associated with $\mathrm{CRC}$ risk (all $p$ for trend $<0.01$ ). When the change in FBG was classified into six trajectory patterns, unfavorable trajectory patterns (high stable and upward) were significantly associated with increased CRC risk (hazard ratio [HR] 2.30, p=0.003; HR 1.19, p=0.007, respectively). In subgroup analyses according to the sex, a significant association between FBG variability (SD or $\mathrm{CV}$ ) and $\mathrm{CRC}$ risk was observed in men but not in women. The high stable and upward pattern were also associated with $C R C$ risk in men (HR 2.47, $p=0.002 ; H R$ 1.21, p=0.012) but not in women.
\end{abstract}

Conclusions: This study identified that FBG variability and unfavorable trajectory patterns were significantly associated with increased $\mathrm{CRC}$ risk in the healthy population without overt diabetes. Our findings suggest that FBG variability as well as FBG itself may be a predictive factor for the development of CRC. (Gut Liver 2022;16:423-432)

Key Words: Blood glucose; Biological variation, individual; Colorectal neoplasms; Cohort study

\section{INTRODUCTION}

Colorectal cancer (CRC) is one of the most frequent malignancies worldwide. According to the reports of the Global Burden of Disease Study, CRC-related mortalities are steadily increasing annually worldwide. ${ }^{1}$ There have been many studies that suggest that the development of CRC is associated with abnormal metabolic conditions, such as obesity, diabetes mellitus (DM), and metabolic syndrome. ${ }^{2-4}$ However, the exact underlying mechanisms still remain unclear.

Meanwhile, DM incidence has been greatly increasing in most countries, including Korea. ${ }^{5,6}$ The mortality rate from DM and its complications are also increasing in Korea. ${ }^{6}$ To date, several studies have investigated the association between DM and CRC. Some studies have shown that $\mathrm{DM}$ is associated with increased risk of CRC or that DM is related to higher CRC-related mortality. ${ }^{7-11}$ Common identified risk factors for both DM and CRC are Westernized diet, obesity, smoking, and lack of physical activity. ${ }^{12}$ Recently, fasting blood glucose (FBG) variability has emerged as a considerable risk factor for long-term diabetic complications. ${ }^{13}$ The Verona Diabetes Study has shown that long-term variability of FBG was significantly predictive of cardiovascular events and mortality in type $2 \mathrm{DM}$ patients who were followed for 10 years. ${ }^{14}$ However, few studies 
have investigated the relationship between FBG variability and cancer risk. ${ }^{14}$

Therefore, we aimed to investigate the association of FBG variability with CRC risk in Korean men and women without overt DM using the nationwide health screening cohort.

\section{MATERIALS AND METHODS}

\section{Data source}

This study was performed using the data from the Korean National Health Insurance Service-Health Screening Cohort (NHIS-Health Screening Cohort), which includes 514,866 Koreans (aged 40 to 79 in 2002) who were randomly selected $10 \%$ among the national health screening participants during 2002 to 2003 . It is a national database established in 2015 by the NHIS. All Koreans have an obligation to join the NHIS, a single health insurance system, and all data related to insurance eligibility and medical claims are stored in the NHIS database. We used data from the general health screening program, which can be applied at least once every 2 years for the aged Korean population (40 years or older). It includes data on demographic variables, bioclinical laboratory results, and questionnaires about risk factors, such as smoking status. NHIS-Health Screening Cohort contains data for all participants followed up until December 31, 2015. Detailed information of the cohort can be found in the cohort profile paper. ${ }^{15}$

\section{Study population}

After excluding subjects with inconsistent date of examination, this study included 514,795 subjects who had taken general health screening at least once between 2002 and 2003. For this study, we excluded subjects who be- long to the following criteria: those with missing data for FBG level at baseline $(n=658)$, those who participated in health screening less than 3 times between 2002 and 2007 $(n=230,778)$, and/or those with a history of inflammatory bowel disease (ICD-10: K50 and K51) (n=3,281) during the entire study period (between 2002 and 2015). Subjects diagnosed with any type of cancer (ICD-10: C00-96) $(\mathrm{n}=8,458)$ and DM (ICD-10: E10-14 and $\geq 1$ claim for antidiabetic medication) $(n=24,684)$ between 2002 and 2007 were excluded. Additionally, subjects who died between 2002 and $2007(n=507)$ or those with glucose measurements less than 3 times between 2002 and $2007(n=188)$ were excluded. Finally, 246,241 subjects were included in this study (Fig. 1).

Between 2002 and 2003, the date of health screening was considered as the index date. Regarding the incident case, the first hospital admission with CRC diagnostic code (ICD-10: C18-20) was defined as the date of CRC occurrence between 2008 and 2015. Thus, the follow-up period was defined from date of the health screening to either the date of CRC diagnosis or the date of the last followup (12/31/2015) if CRC did not develop. This study was approved by the Institutional Review Board of the Ewha Womans University Mokdong Hospital (IRB number: EUMC 2018-12-007). Informed consent was waived due to the retrospective design of this study.

\section{Variability definition}

We defined FBG variability using repeatedly measured FBG data for each individual between 2002 and 2007. FBG variability was calculated using standard deviation (SD) and coefficient of variation (CV). The variability was divided into quintiles and analyzed: quintiles of SD (Q1, <4.97; Q2, 4.97-7.49; Q3, 7.50-10.11; Q4, 10.12-14.19; and $\mathrm{Q} 5, \geq 14.20)$ and quintiles of CV (Q1, <5.45; Q2, 5.45-8.18;

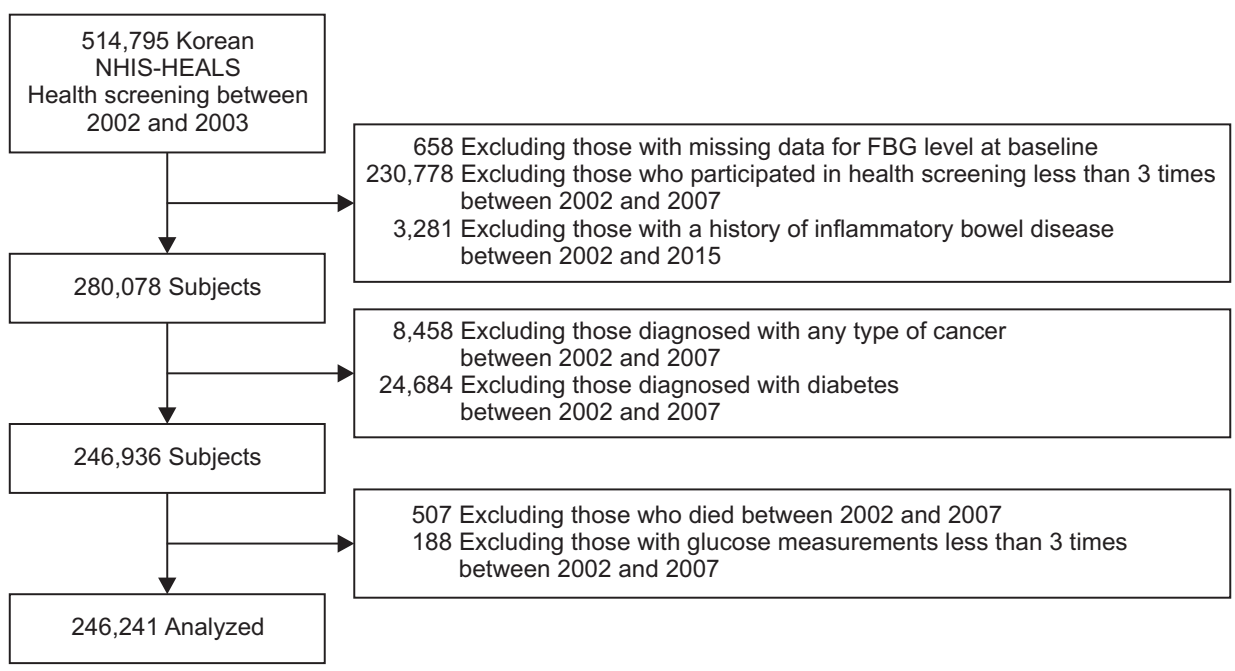

Fig. 1. Flow diagram of the study subjects.

NHIS-HEALS, National Health Insurance Service-Health Screening Cohort; FBG, fasting blood glucose. 
Q3, 8.19-11.08; Q4, 11.09-15.26; and Q5, 215.27). Additionally, we defined the trajectory patterns using the measured values in the years 2002-2003 (T1), 2004-2005 (T2), and 2006-2007 (T3). FBG concentrations at each time point were divided into three levels using cutoff values of $100 \mathrm{mg} / \mathrm{dL}$ and $126 \mathrm{mg} / \mathrm{dL}$, and then, six trajectory patterns were formed using the change of fasting glucose status (Supplementary Table 1, Supplementary Fig. 1). The NHIS-Health Screening Cohort provides income level data as medical aid beneficiaries, deciles for insured employees, and deciles for insured self-employed based on incomebased insurance contributions. Thus, we classified medical aid beneficiaries and the first and second deciles into one group. Sequentially, the two deciles were grouped and applied to the analysis.

\section{Statistical analyses}

Descriptive results were summarized as means with SD for numerical data and as number of subjects with percentage for categorical data. The incidence rate of CRC was estimated as 100,000 person-years. The risk of developing CRC according to FBG variability was estimated using the Cox proportional hazard model analyses, and the results were presented as hazard ratio (HR) with 95\% confidence interval (CI). Its assumption was tested by the Schoenfeld residuals, and the assump- tion was satisfied. We considered age, income level, body mass index status $\left(<20 \mathrm{~kg} / \mathrm{m}^{2}, 20-24.9 \mathrm{~kg} / \mathrm{m}^{2}\right.$, and $\geq 25 \mathrm{~kg} /$ $\mathrm{m}^{2}$ ), smoking (never, former, and current smoker), alcohol intake (never, 2-3 times/month, 1-2 times/week, and $\geq 3$ times/ week), physical activity (none, $1-2$ times/week, and $\geq 3$ times/ week), and Charlson comorbidity index (CCI) as confounding variables. Sensitivity analysis was performed by excluding subjects who had developed CRC within 2 years by adjusting various covariates. In addition, to visually assess the shape of association between FBG variability and incident CRC, restricted cubic spline was used. Thus, based on the low Akaike information criterion values, Cox models with restricted cubic spline were conducted using 3 knots at the 10th, 50th, and 90th percentiles. All statistical analyses were conducted using SAS version 9.4 (SAS Institute, Cary, NC, USA), and $\mathrm{p}<0.05$ was considered significant under two-sided test.

\section{RESULTS}

\section{Baseline characteristics of the study population}

The study was analyzed on a total of 246,241 subjects, 143,623 men and 102,618 women. The mean age of the study population was $51.20 \pm 8.81$ years $(50.44 \pm 8.64$ years in men, $52.26 \pm 8.95$ in women) (Table 1). With regard to

Table 1. Baseline Characteristics of the Study Subjects

\begin{tabular}{|c|c|c|c|c|}
\hline Characteristics & & $\begin{array}{c}\text { Total } \\
\text { (n=246,241) }\end{array}$ & $\begin{array}{c}\text { Men } \\
\text { (n=143,623) }\end{array}$ & $\begin{array}{c}\text { Women } \\
(n=102,618)\end{array}$ \\
\hline Age, mean $\pm S D$, yr & & $51.20 \pm 8.81$ & $50.44 \pm 8.64$ & $52.26 \pm 8.95$ \\
\hline \multirow[t]{5}{*}{ Income level, No. (\%)* } & Medical aid beneficiaries $+\leq 20$ th & $37,455(15.21)$ & 15,407 (10.73) & 22,048 (21.49) \\
\hline & $21-40$ th & 31,994 (12.99) & 15,193 (10.58) & $16,801(16.37)$ \\
\hline & 41-60th & 37,004 (15.03) & $21,916(15.26)$ & $15,088(14.70)$ \\
\hline & 61-80th & $50,683(20.58)$ & $32,455(22.60)$ & $18,228(17.76)$ \\
\hline & $\geq 81$ th & 89,105 (36.19) & $58,652(40.84)$ & 30,453 (29.68) \\
\hline \multirow[t]{3}{*}{ Body mass index, No. (\%) } & $<20 \mathrm{~kg} / \mathrm{m}^{2}$ & $19,194(7.80)$ & $10,531(7.34)$ & $8,663(8.45)$ \\
\hline & $20-24.9 \mathrm{~kg} / \mathrm{m}^{2}$ & $145,825(59.26)$ & 84,103 (58.59) & 61,722 (60.19) \\
\hline & $\geq 25 \mathrm{~kg} / \mathrm{m}^{2}$ & 81,053 (32.94) & 48,899 (34.07) & $32,154(31.36)$ \\
\hline \multirow[t]{3}{*}{ Smoking, No. $(\%)$} & Never & $160,867(66.45)$ & $61,071(43.69)$ & 99,796 (97.54) \\
\hline & Previous & $23,397(9.66)$ & $22,610(16.17)$ & $787(0.77)$ \\
\hline & Current & 57,840 (23.89) & $56,105(40.14)$ & $1,735(1.70)$ \\
\hline \multirow[t]{4}{*}{ Alcohol intake, No. (\%) } & Never & $128,654(53.19)$ & 46,447 (32.78) & 82,207 (82.03) \\
\hline & 2-3 times/mo & 41,834 (17.29) & $30,609(21.61)$ & $11,225(11.20)$ \\
\hline & $1-2$ times/wk & $45,160(18.67)$ & $39,958(28.20)$ & $5,202(5.19)$ \\
\hline & $\geq 3$ times/wk & $26,244(10.85)$ & $24,659(17.41)$ & $1,585(1.58)$ \\
\hline \multirow[t]{3}{*}{ Physical activity, No. (\%) } & None & $130,023(54.39)$ & $64,469(46.30)$ & $65,554(65.68)$ \\
\hline & 1-2 times/wk & $63,578(26.60)$ & $45,763(32.86)$ & 17,815 (17.85) \\
\hline & $\geq 3$ times/wk & 45,453 (19.01) & $29,020(20.84)$ & $16,433(16.47)$ \\
\hline $\mathrm{CCl}$ score, mean $\pm \mathrm{SD}$ & & $0.41 \pm 0.75$ & $0.35 \pm 0.70$ & $0.51 \pm 0.81$ \\
\hline $\mathrm{FBG}$, mean $\pm \mathrm{SD}, \mathrm{mg} / \mathrm{dL}$ & & $92.70 \pm 23.93$ & $93.88 \pm 23.16$ & $91.06 \pm 24.87$ \\
\hline $\mathrm{SD}$ of $\mathrm{FBG}$, mean $\pm \mathrm{SD}$ & & $10.78 \pm 13.53$ & $11.44 \pm 13.12$ & $9.85 \pm 14.02$ \\
\hline $\mathrm{CV}$ of $\mathrm{FBG}$, mean $\pm \mathrm{SD}$ & & $11.04 \pm 7.78$ & $11.56 \pm 7.98$ & $10.31 \pm 7.42$ \\
\hline
\end{tabular}

$\mathrm{CCl}$, Charlson comorbidity index; FBG, fasting blood glucose; SD, standard deviation; CV, coefficient of variation.

*Income level: The National Health Insurance Service-Health Screening Cohort provides income level data as medical aid beneficiaries, deciles for insured employees, and deciles for insured self-employed individuals. 
body mass index, more women were underweight $(<20$ $\left.\mathrm{kg} / \mathrm{m}^{2}\right)$ than men $(8.45 \%$ in women, $7.34 \%$ in men). Conversely, more men were overweight $\left(\geq 25 \mathrm{~kg} / \mathrm{m}^{2}\right)$ than women (34.07\% in men, $31.36 \%$ in women). Current smokers among men were $40.14 \%$, being approximately 40 times as many as women. The proportion of individuals consuming alcohol more than 3 times a week was much higher in men (17.41\%) than in women (1.58\%). However, more women did not exercise any physical activity during the week (65.68\%) than men (46.30\%). The average of FBG level at baseline was higher in men $(93.88 \pm 23.16 \mathrm{mg} / \mathrm{dL})$ than in women $(91.05 \pm 24.87 \mathrm{mg} / \mathrm{dL})$, and the indicators of variability, $\mathrm{SD}$ and $\mathrm{CV}$, were also higher in men.

\section{Association of FBG variability with $\mathrm{CRC}$ risk}

The incidence of CRC during the follow-up period was 116.7 per 100,000 person-years $(n=3,669)$. We evaluated the effect of FBG variability on CRC risk using the Cox proportional hazard regression analysis (Table 2). To analyze the association of FBG variability, SD or $\mathrm{CV}$, with CRC incidence, model 1 was adjusted for the age and sex in the crude model, and model 2 was adjusted for various confounding factors (economic income, body mass index, smoking habits, alcohol intake, physical activity, and CCI) in model 1. The highest quintile of SD showed a significantly higher risk of incident CRC (HR, 1.41; 95\% CI, 1.27 to 1.56 ) compared with the lowest quintile of SD. The significance remained after adjusting for various covariates (HR, 1.19; 95\% CI, 1.07 to 1.32). When SD of FBG was divided into five quintiles, an increase in the quintile of SD was significantly associated with increased risk of CRC in the crude model ( $\mathrm{p}$ for trend $<0.001$ ), model 1 ( $\mathrm{p}$ for trend $<0.001$ ), and model 2 ( $\mathrm{p}$ for trend=0.001). An increase in the quintile of $\mathrm{CV}$ was also associated with CRC risk in the crude model ( $\mathrm{p}$ for trend $<0.001$ ), model 1 ( $\mathrm{p}$ for trend $=0.001$ ), and model 2 ( $\mathrm{p}$ for trend $=0.009$ ). In the sensitivity analysis excluding subjects who developed CRC within 2 years, the influence of the SD or CV FBG variability on CRC incidence remained as shown in Table 2 and Supplementary Table 2. Among the confounding variables, male, age, current smoking status, high intake of alcohol, high body mass index and CCI were independently associated with CRC (Supplementary Table 3). For CRC, there was no interactive effect between FBG variability and confounding variables (Supplementary Table 4). Even when separately evaluated for rectal cancer, the effect of the variability evaluated by SD and CV was similar to the above results (Supplementary Table 5). After the median value of FBG variability assessed by SD or CV, the risk of CRC gradually increased as the variability increased, but the CV-derived results showed a wider CI (Fig. 2A and B).

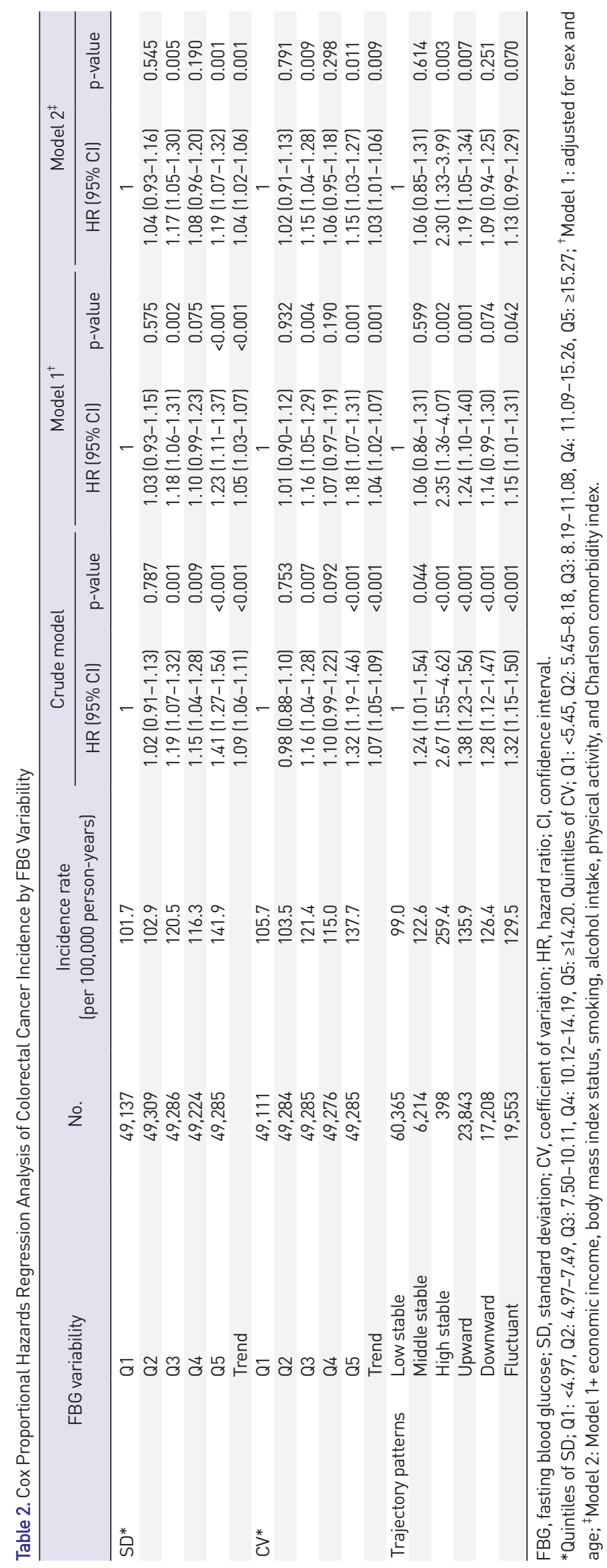


When the change of FBG level was classified into six trajectory patterns, unfavorable patterns, namely high stable, upward, and fluctuant patterns showed a significantly increased risk of CRC in model 1 (HR 2.35, $\mathrm{p}=0.002$; HR $1.24, \mathrm{p}=0.001$; HR $1.15, \mathrm{p}=0.042$, respectively). In model 2 , high stable and upward trajectory patterns were significantly associated with increased CRC risk (HR 2.30, $\mathrm{p}=0.003$; HR 1.19, $\mathrm{p}=0.007$, respectively) (Table 2). However, the association of the fluctuant pattern with CRC risk did not reach statistical significance in model 2 (HR 1.13, $\mathrm{p}=0.070$ ). In the sensitivity analysis, a high stable trajectory pattern was still significantly associated with an increased risk of CRC, and an upward trajectory pattern was associated with a borderline significance level (Supplementary Table 2).

\section{Association of FBG variability with CRC risk among men and women}

During the 8-year follow-up (between 2008 and 2015), the incidence of CRC was 135.2 per 100,000 person-years $(n=2,487)$ in men and 90.5 per 100,000 person-years $(n=1,182)$ in women. To evaluate whether the association between FBG variability and CRC incidence differed according to the sex of the subjects, univariate (crude model) and multivariate analyses (model 1 and model 2) were carried out for male and female subjects. In men, an increase in the quintile of SD was significantly associated with increased risk of CRC in the crude model ( $\mathrm{p}$ for trend $<0.001$ ), model 1 ( $\mathrm{p}$ for trend $<0.001$ ), and model 2 ( $\mathrm{p}$ for trend=0.007). An increase in the quintile of $\mathrm{CV}$ was also associated with increased CRC risk in the crude model ( $\mathrm{p}$ for trend $<0.001$ ), model 1 ( $\mathrm{p}$ for trend=0.001), and model 2 ( $\mathrm{p}$ for trend $=0.027$ ). With regard to trajectory patterns, unfavorable patterns (high stable, upward, and fluctuant patterns) were significantly associated with increased CRC risk in the crude model. Compared with the low stable trajectory pattern, high stable and upward patterns were shown to significantly increase the risk of CRC in model 1 (HR 2.54, p=0.001; HR 1.27, p=0.001, respectively). Even after adding various covariates (model 2 ),
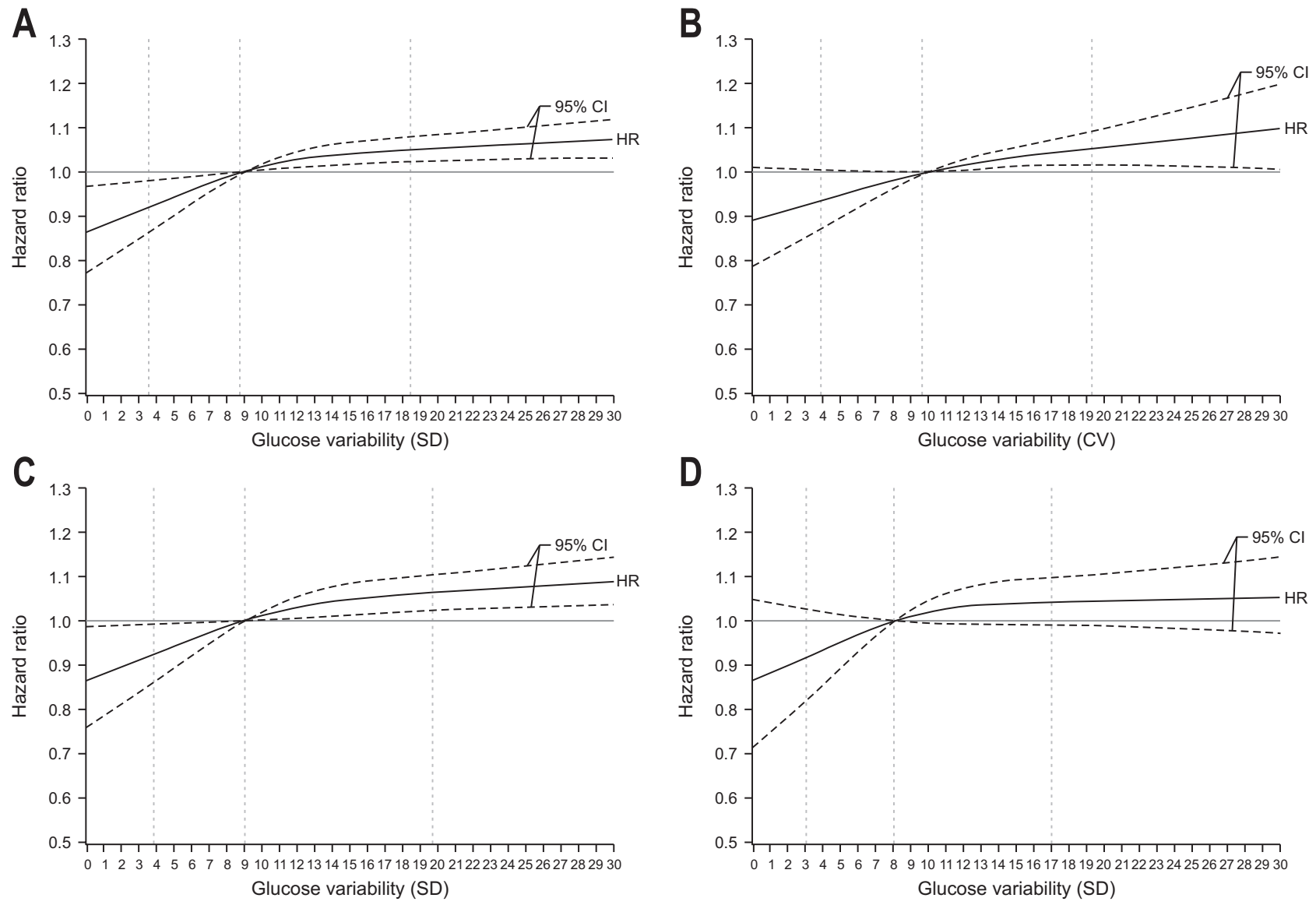

Fig. 2. Association of FBG variability assessed by SD (A) or CV (B) with incident $C R C$ risk and association of FBG variability assessed by SD with incident CRC risk in men (C) and women (D). The solid line indicates the HR, and dashed lines indicate the $95 \% \mathrm{Cl}$. The model was fitted by a restricted cubic spline with three knots located at the 10th, 50th, and 90th percentiles of the FBG variability (vertical lines). HRs with $95 \%$ Cls were obtained after adjusting for sex, age, income, body mass index status, smoking habits, alcohol intake, physical activity, and the Charlson comorbidity index. FBG, fasting blood glucose; SD, standard deviation; CV, coefficient of variation; $\mathrm{CRC}$, colorectal cancer; $\mathrm{HR}$, hazard ratio; $\mathrm{Cl}$, confidence interval. 
the high stable and upward pattern was significantly associated with increased CRC risk (HR 2.47, p=0.002; HR 1.21, $\mathrm{p}=0.012$, respectively) (Table 3 ). Among the confounding variables, age, income level, current smoking, and high intake of alcohol were independently associated with CRC (Supplementary Table 6). In the sensitivity analysis, high stability and upward patterns still showed a significant association for CRC risk (Supplementary Table 2). In addition, a positive association between rectal cancer with SD or $\mathrm{CV}$ of FBG was significant, and the risk of rectal cancer was significantly higher in the high-stable group compared to the low-stable group (HR 2.59, $\mathrm{p}=0.037$ ) (Supplementary Table 5).

In women, a significant association was observed between the quintile of SD and CV and CRC incidence in the crude model ( $\mathrm{p}$ for trend $=0.001$ and $\mathrm{p}$ for trend $=0.014$, respectively); however, this association did not reach statistrend $=0.264$, respectively) and model 2 ( $\mathrm{p}$ for trend $=0.067$ and $\mathrm{p}$ for trend $=0.162$, respectively). In addition, there was also no significant difference in the sensitivity analysis (Supplementary Table 2). Similarly, regarding trajectory patterns, there was no significant difference in CRC risk among the trajectory patterns in models 1 and 2 among women (Table 4). Among the confounding variables, age, physical activity level, and CCI score were independently associated with CRC (Supplementary Table 7). The risk of rectal cancer according to FBG variability was also insignificant (Supplementary Table 5). In both sexes, the risk of CRC gradually increased after the median value of FBG variability assessed by SD. However, a statistical significance was observed in men, but not in women (Fig. 2C and D).

\section{DISCUSSION}

In the comprehensive health screening cohort in Korean men and women excluding patients with overt DM, we investigated the association of FBG variability with CRC risk. Multivariate analyses adjusted for several covariates found that FBG variability, represented by SD or CV, was significantly associated with increased CRC risk. Regarding FBG trajectory patterns, unfavorable patterns (high stable and upward) were positively associated with increased CRC risk.

Various previous studies have reported that increased FBG level is related with CRC incidence. It is well-known that DM is significantly associated with the increased risk of CRC., ${ }^{4,12,16}$ Type $2 \mathrm{DM}$, in particular, increases the risk of CRC by 1.3 times $^{17}$ and is an independent risk factor for tical significance in model 1 ( $\mathrm{p}$ for trend $=0.099$ and $\mathrm{p}$ for

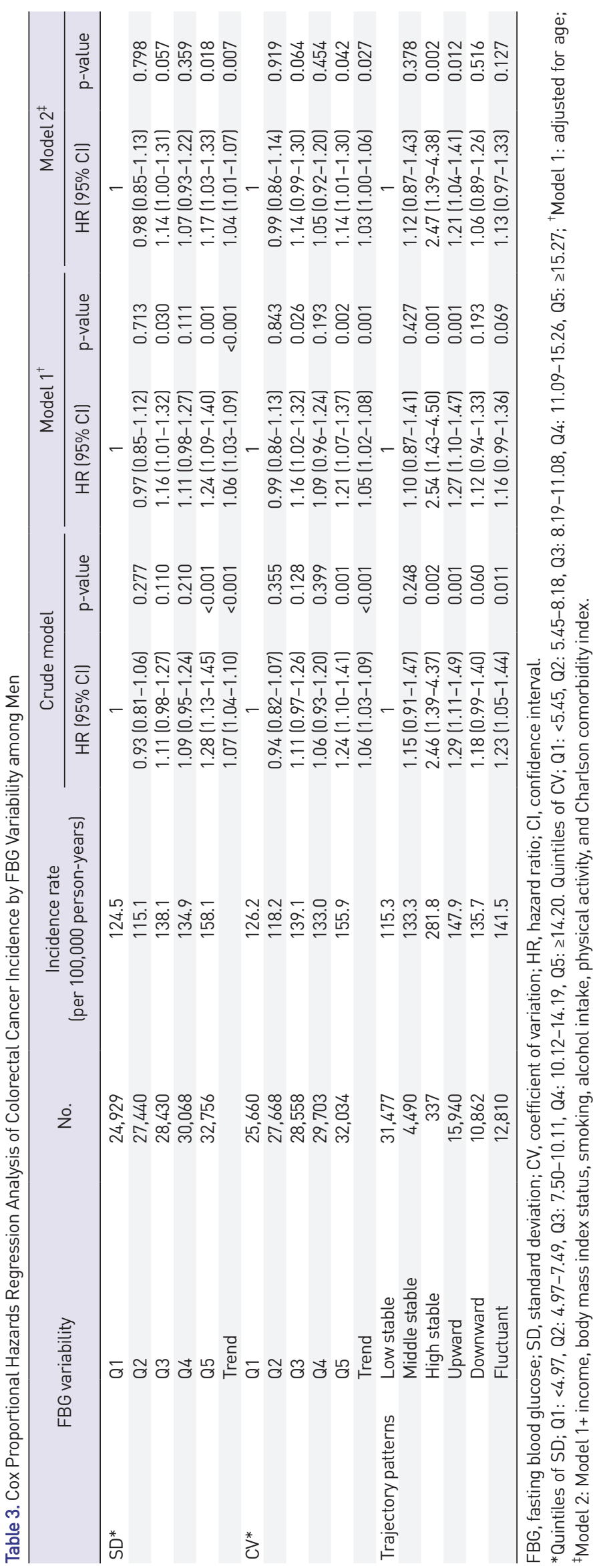




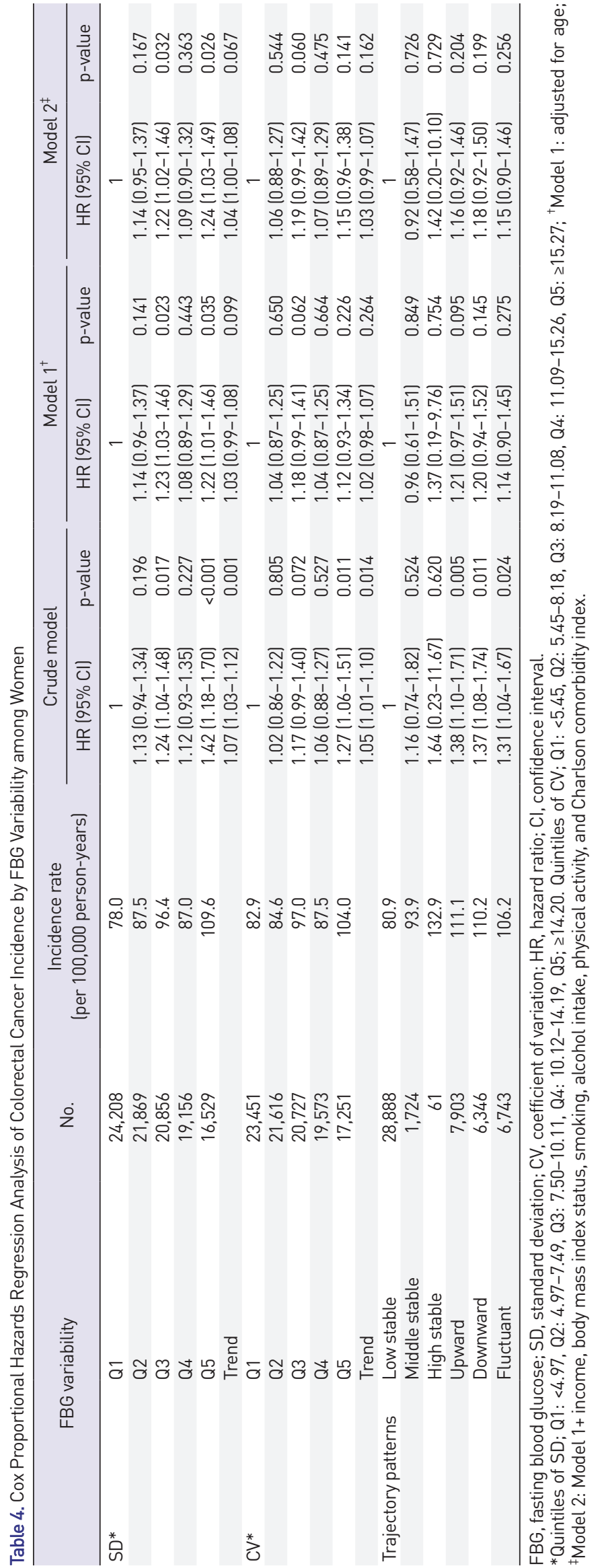

mortality of several solid cancers, including colon cancer. ${ }^{18}$ In the Korean Cancer Prevention Study-II, a large-scale prospective cohort study, CRC incidence was positively associated with increased FBG levels. ${ }^{19}$ The Framingham Offspring Cohort study has also shown that the presence of impaired fasting glucose $>10$ years before the event was correlated with increased risk of obesity-related cancers, particularly CRC. ${ }^{20}$ The association between increased FBG and CRC has been explained by several hypotheses. Hyperinsulinemia, ${ }^{21,22}$ hyperglycemia, ${ }^{22}$ or associated cofactors are associated with increased risk of CRC. Hyperinsulinemia through insulin-like growth factor system ${ }^{23-25}$ can influence the differentiation of epithelial cells in the intestine and can contribute to colorectal neoplasia. ${ }^{26}$ Other factors, such as chronic inflammation, ${ }^{27,28}$ imbalanced intestinal microbiota, ${ }^{29}$ or delayed bowel transit, ${ }^{30}$ have also been proposed.

Meanwhile, the clinical significance of FBG variability was found to be associated with diabetic complications or mortality. In the Verona Diabetes Study, long-term variability of FBG was significantly predictive of cardiovascular events and mortality in type $2 \mathrm{DM}$ patients followed up for 10 years. ${ }^{14}$ Recently, a meta-analysis investigated the association of FBG variability with complications and mortality in five studies including patients with type 2 DM. ${ }^{31}$ This analysis reported that high FBG variability was significantly associated with the risk of retinopathy (odds ratio, 3.68; $95 \%$ CI, 1.01 to 13.4 ) and all-cause mortality (HR, 1.28; 95\% CI, 1.12 to 1.46). In Korea, one prospective cohort study (Ansung-Ansan cohort) has investigated the relationship between glucose variability and vascular adverse events in middle-aged individuals without DM at baseline. ${ }^{32}$ This study reported that higher FBG variability and post 2-hour blood glucose were predictive factors for macrovascular events, including coronary artery disease, myocardial infarction, and congestive heart failure and stroke but not for microvascular events. ${ }^{32}$ With regard to the underlying mechanisms, FBG variability may be associated with oxidative stress, inflammatory cytokine production, and some epigenetic changes such as cellular memory. ${ }^{33-37}$

Thus far, few studies have evaluated the association between FBG variability and cancer risk. The Verona Diabetes Study identified that high FBG variability (represented by the CV value) was the risk factor of mortality from malignancies as well as all-cause or cardiovascular-related mortalities. ${ }^{14}$ Although this association may be explained by the deterioration of FBG over time in individuals with high CV of FBG, it has not been supported by any clinical or experimental studies. Similar to the Verona Diabetes Study, our study reports a significant association between 
high CV (or SD) of FBG and CRC risk. In addition, high stable and upward FBG trajectory patterns were independent predictors of CRC incidence. Among six FBG trajectory patterns in our study, high stable, upward, and fluctuant groups could be classified as unfavorable patterns considering the long-term outcomes related to DM. Although the fluctuant FBG trajectory pattern tended to be associated with the risk of CRC, it did not reach statistical significance. To verify and confirm our results, further large-scale studies across different ethnic populations are warranted.

In this study, we also evaluated the association between FBG variability and CRC risk according to the sex. Consequently, SD or CV of FBG was significantly associated with the risk of CRC in men but not in women. Some unfavorable trajectory patterns were also significantly associated with CRC risk in men (high stable and upward patterns) but not in women. These results are similar to the CRC risk in DM patients according to the sex. In the Cancer Prevention Study II Nutrition Cohort, a significant association between type $2 \mathrm{DM}$ and CRC incidence was found among men but not women. ${ }^{38}$ In addition, the Korean Cancer Prevention Study-II, a prospective cohort study of a large Korean population, showed that high FBG $(\geq 100 \mathrm{mg} / \mathrm{dL})$ including impaired fasting glucose correlated with the risk of CRC only in men. ${ }^{19}$ When analyzed according to the sex, the risk associated with developing CRC in study subjects with high FBG was statistically significant in men (HR, 1.51; 95\% CI, 1.11 to 2.05), whereas no such association was noted in women (HR, 1.23 ; 95\% CI, 0.67 to 2.26 ). ${ }^{19}$ Another Korean cohort study has also reported that the association of impaired fasting glucose with CRC risk was observed only in men. ${ }^{39}$ Taken together, our results suggest that men are more susceptible to CRC than women if they have FBG variability or some unfavorable trajectory patterns.

The present study has some limitations. First, as this was a retrospective study, our results may have been influenced by the accuracy and availability of the medical records. In particular, a considerable number of subjects $(n=230,778)$ were excluded if they participated in health screening less than 3 times between 2002 and 2007. This may have led to selection bias and caused the insufficient sample size in the specific groups to evaluate the association between FBG variability and CRC risk. Second, since the major outcome in our study was CRC, there may be a length time bias when considering the limited period of this study. Third, we did not reflect all the changes in various factors (drugs, health behaviors, etc.) during the FBG variability assessment period. However, when the CCI calculated using the claims data for 2002 to 2007 was applied, the results did not change (data not shown). Fourth, regarding FBG trajectory patterns, the high stable group had much fewer cases than other groups. We excluded subjects diagnosed with DM between 2002 and 2007. DM was defined as prescribing one or more antidiabetic medications along with E10-14 in the ICD-10 code in our study. Accordingly, if any individual with FBG levels $\geq 126 \mathrm{mg} / \mathrm{dL} 3$ times was diagnosed with DM and was prescribed antidiabetic medications, they were excluded from our study. However, despite the small sample size, the high stable trajectory pattern was independently associated with the CRC risk in the overall and male subjects. Fifth, there was lack of information or separate diagnostic code for family history of CRC or hereditary CRC syndrome in our cohort data from the Korean NHIS database. Thus, we could not exclude those with hereditary CRC syndrome or with a family history of CRC. In addition, we could not include the colonoscopy performance and polypectomy history in our study. Thus, further studies to conduct with more organized algorithm were needed to verify our result. Despite these limitations, it is meaningful that this study is the first to evaluate the association between the various indicators of FBG variability and CRC risk and to find its sex differences.

In conclusion, in this study, we found that FBG variability and the unfavorable trajectory patterns - high stable and upward-were significantly associated with increased $\mathrm{CRC}$ risk in the healthy population without overt DM. The association of FBG variability and the unfavorable patterns with CRC risk persisted in men, but not in women. Thus, our findings suggest that FBG variability as well as FBG itself may be a predictive factor for the development of CRC. Further researches on FBG variability in a healthy population may be used to individually tailor a CRC screening strategy.

\section{CONFLICTS OF INTEREST}

No potential conflict of interest relevant to this article was reported.

\section{ACKNOWLEDGEMENTS}

This work was supported by the National Research Foundation of Korea (NRF) grant funded by the Korea government (MSIT) (2020R1A2C1010786 and 2020R1A5A2019210; C.M.M.).

C.M.M. had full access to all of the data in the study and takes responsibility for the integrity of the data and the accuracy of the data analysis. 


\section{AUTHOR CONTRIBUTIONS}

Concept and design: C.M.M., H.A.L. Acquisition, analysis, or interpretation of data: C.M.M., H.J., J. L., H.A.L., S.E.K., K.N.S., H.K.J., S.A.J. Statistical analysis: H.A.L. Obtained funding: C.M.M. Administrative, technical, or material support: C.M.M., H.A.L. Supervision: C.M.M., H.A.L. Drafting of the manuscript: C.M.M., H.A.L., H.J., J.L. Critical revision of the manuscript for important intellectual content: C.M.M., H.A.L., S.E.K., K.N.S., H.K.J., S.A.J.

\section{ORCID}

Hyoju Jun https://orcid.org/0000-0001-7042-8756

Jieun Lee https://orcid.org/0000-0002-6256-3947

Hye Ah Lee https://orcid.org/0000-0002-4051-0350

Seong-Eun Kim https://orcid.org/0000-0002-6310-5366

Ki-Nam Shim https://orcid.org/0000-0003-4004-6292

Hye-Kyung Jung https://orcid.org/0000-0002-6653-5214

Sung-Ae Jung https://orcid.org/0000-0001-7224-2867

Chang Mo Moon https://orcid.org/0000-0003-2550-913X

\section{SUPPLEMENTARY MATERIALS}

Supplementary materials can be accessed at https://doi. org/10.5009/gnl210048.

\section{REFERENCES}

1. GBD 2013 Mortality and Causes of Death Collaborators. Global, regional, and national age-sex specific all-cause and cause-specific mortality for 240 causes of death, 1990-2013: a systematic analysis for the Global Burden of Disease Study 2013. Lancet 2015;385:117-171.

2. Jee SH, Yun JE, Park EJ, et al. Body mass index and cancer risk in Korean men and women. Int J Cancer 2008;123:18921896.

3. Ishino K, Mutoh M, Totsuka Y, Nakagama H. Metabolic syndrome: a novel high-risk state for colorectal cancer. Cancer Lett 2013;334:56-61.

4. Giovannucci E, Harlan DM, Archer MC, et al. Diabetes and cancer: a consensus report. Diabetes Care 2010;33:16741685.

5. Shaw JE, Sicree RA, Zimmet PZ. Global estimates of the prevalence of diabetes for 2010 and 2030. Diabetes Res Clin Pract 2010;87:4-14.

6. Kim DJ. The epidemiology of diabetes in Korea. Diabetes Metab J 2011;35:303-308.
7. Rosato V, Tavani A, Gracia-Lavedan E, et al. Type 2 diabetes, antidiabetic medications, and colorectal cancer risk: two case-control studies from Italy and Spain. Front Oncol 2016;6:210.

8. de Kort S, Simons CC, van den Brandt PA, et al. Diabetes mellitus type 2 and subsite-specific colorectal cancer risk in men and women: results from the Netherlands Cohort Study on diet and cancer. Eur J Gastroenterol Hepatol 2016;28:896903.

9. Zhu B, Wu X, Wu B, Pei D, Zhang L, Wei L. The relationship between diabetes and colorectal cancer prognosis: a meta-analysis based on the cohort studies. PLoS One 2017;12:e0176068.

10. Mills KT, Bellows CF, Hoffman AE, Kelly TN, Gagliardi G. Diabetes mellitus and colorectal cancer prognosis: a metaanalysis. Dis Colon Rectum 2013;56:1304-1319.

11. Luo J, Lin HC, He K, Hendryx M. Diabetes and prognosis in older persons with colorectal cancer. Br J Cancer 2014;110:1847-1854.

12. Deng L, Gui Z, Zhao L, Wang J, Shen L. Diabetes mellitus and the incidence of colorectal cancer: an updated systematic review and meta-analysis. Dig Dis Sci 2012;57:1576-1585.

13. Smith-Palmer J, Brändle M, Trevisan R, Orsini Federici $\mathrm{M}$, Liabat S, Valentine W. Assessment of the association between glycemic variability and diabetes-related complications in type 1 and type 2 diabetes. Diabetes Res Clin Pract 2014;105:273-284.

14. Muggeo M, Zoppini G, Bonora E, et al. Fasting plasma glucose variability predicts 10 -year survival of type 2 diabetic patients: the Verona Diabetes Study. Diabetes Care 2000;23:45-50.

15. Seong SC, Kim YY, Park SK, et al. Cohort profile: the National Health Insurance Service-National Health Screening Cohort (NHIS-HEALS) in Korea. BMJ Open 2017;7:e016640.

16. Larsson SC, Orsini N, Wolk A. Diabetes mellitus and risk of colorectal cancer: a meta-analysis. J Natl Cancer Inst 2005;97:1679-1687.

17. Peeters PJ, Bazelier MT, Leufkens HG, de Vries F, De Bruin ML. The risk of colorectal cancer in patients with type 2 diabetes: associations with treatment stage and obesity. Diabetes Care 2015;38:495-502.

18. Coughlin SS, Calle EE, Teras LR, Petrelli J, Thun MJ. Diabetes mellitus as a predictor of cancer mortality in a large cohort of US adults. Am J Epidemiol 2004;159:1160-1167.

19. Shin HY, Jung KJ, Linton JA, Jee SH. Association between fasting serum glucose levels and incidence of colorectal cancer in Korean men: the Korean Cancer Prevention Study-II. Metabolism 2014;63:1250-1256.

20. Parekh N, Lin Y, Vadiveloo M, Hayes RB, Lu-Yao GL. Metabolic dysregulation of the insulin-glucose axis and risk of obesity-related cancers in the Framingham heart study- 
offspring cohort (1971-2008). Cancer Epidemiol Biomarkers Prev 2013;22:1825-1836.

21. Giovannucci E. Insulin and colon cancer. Cancer Causes Control 1995;6:164-179.

22. McKeown-Eyssen G. Epidemiology of colorectal cancer revisited: are serum triglycerides and/or plasma glucose associated with risk? Cancer Epidemiol Biomarkers Prev 1994;3:687-695.

23. Ish-Shalom D, Christoffersen CT, Vorwerk P, et al. Mitogenic properties of insulin and insulin analogues mediated by the insulin receptor. Diabetologia 1997;40 Suppl 2:S25-S31.

24. Khandwala HM, McCutcheon IE, Flyvbjerg A, Friend KE. The effects of insulin-like growth factors on tumorigenesis and neoplastic growth. Endocr Rev 2000;21:215-244.

25. Cohen DH, LeRoith D. Obesity, type 2 diabetes, and cancer: the insulin and IGF connection. Endocr Relat Cancer 2012;19:F27-F45.

26. Tran TT, Naigamwalla D, Oprescu AI, et al. Hyperinsulinemia, but not other factors associated with insulin resistance, acutely enhances colorectal epithelial proliferation in vivo. Endocrinology 2006;147:1830-1837.

27. Yu H, Pardoll D, Jove R. STATs in cancer inflammation and immunity: a leading role for STAT3. Nat Rev Cancer 2009;9:798-809.

28. Straus DS. TNFa and IL-17 cooperatively stimulate glucose metabolism and growth factor production in human colorectal cancer cells. Mol Cancer 2013;12:78.

29. Tlaskalová-Hogenová $H$, Stěpánková $R$, Kozáková $H$, et al. The role of gut microbiota (commensal bacteria) and the mucosal barrier in the pathogenesis of inflammatory and autoimmune diseases and cancer: contribution of germ-free and gnotobiotic animal models of human diseases. Cell Mol Immunol 2011;8:110-120.

30. Will JC, Galuska DA, Vinicor F, Calle EE. Colorectal cancer: another complication of diabetes mellitus? Am J Epidemiol
1998;147:816-825.

31. Zhao Q, Zhou F, Zhang Y, Zhou X, Ying C. Fasting plasma glucose variability levels and risk of adverse outcomes among patients with type 2 diabetes: a systematic review and meta-analysis. Diabetes Res Clin Pract 2019;148:23-31.

32. Jang JY, Moon S, Cho S, Cho KH, Oh CM. Visit-to-visit $\mathrm{HbAlc}$ and glucose variability and the risks of macrovascular and microvascular events in the general population. Sci Rep 2019;9:1374.

33. Ceriello A, Esposito K, Piconi L, et al. Oscillating glucose is more deleterious to endothelial function and oxidative stress than mean glucose in normal and type 2 diabetic patients. Diabetes 2008;57:1349-1354.

34. Quagliaro L, Piconi L, Assaloni R, Martinelli L, Motz E, Ceriello A. Intermittent high glucose enhances apoptosis related to oxidative stress in human umbilical vein endothelial cells: the role of protein kinase $\mathrm{C}$ and $\mathrm{NAD}(\mathrm{P}) \mathrm{H}$-oxidase activation. Diabetes 2003;52:2795-2804.

35. Schisano B, Tripathi G, McGee K, McTernan PG, Ceriello A. Glucose oscillations, more than constant high glucose, induce p53 activation and a metabolic memory in human endothelial cells. Diabetologia 2011;54:1219-1226.

36. Keating ST, El-Osta A. Glycemic memories and the epigenetic component of diabetic nephropathy. Curr Diab Rep 2013;13:574-581.

37. Sun B, Luo Z, Zhou J. Comprehensive elaboration of glycemic variability in diabetic macrovascular and microvascular complications. Cardiovasc Diabetol 2021;20:9.

38. Campbell PT, Deka A, Jacobs EJ, et al. Prospective study reveals associations between colorectal cancer and type 2 diabetes mellitus or insulin use in men. Gastroenterology 2010;139:1138-1146.

39. Jee SH, Ohrr H, Sull JW, Yun JE, Ji M, Samet JM. Fasting serum glucose level and cancer risk in Korean men and women. JAMA 2005;293:194-202. 